\title{
A new integrated photosensor for gas proportional scintillation counters based on the gas electron multiplier (GEM)
}

\author{
J.A.M. Lopes ${ }^{\mathrm{a}, \mathrm{b}}$, J.M.F. dos Santos ${ }^{\mathrm{a}}$, C.A.N. Conde ${ }^{\mathrm{a}, *}$, R.E. Morgado ${ }^{\mathrm{c}}$ \\ ${ }^{a}$ Departamento de Física, Universidade de Coimbra, P-3000 Coimbra, Portugal \\ ${ }^{\mathrm{b}}$ Instituto Superior de Engenharia de Coimbra, P-3000 Coimbra, Portugal \\ ${ }^{\mathrm{c}}$ Los Alamos National Laboratory, Los Alamos, New Mexico, USA
}

Received 20 July 1998; received in revised form 9 November 1998

\begin{abstract}
The performance of a novel integrated photosensor for use in a xenon gas proportional scintillation detector is described. Earlier integrated photo-sensor designs were limited in charge gains due to the onset of electrical breakdown, which was ascribed to optical positive feedback from scintillation photons produced in the charge amplification stage. The present design uses a gas electron multiplier (GEM) composed of a $50 \mu \mathrm{m}$ thick Kapton film with copper-plated electrode surfaces on both sides and perforated with $200 \mu \mathrm{m}$ holes at a $300 \mu \mathrm{m}$ pitch. The front surface is made photosensitive with a 150-nm-thick CsI film. When an appropriate voltage is applied between the copper electrodes, the resulting electric field directs photoelectrons produced on the front surface through the holes in the GEM and onto a wire chamber where charge amplification occurs. Optical positive feedback is essentially eliminated since the charge amplification stage is optically de-coupled from the photocathode. The GEM also provides a small amount of charge gain, up to 3.3 , before the electrons enter the wire chamber where charge gains up to about $10^{3}$ take place. However, the measured effective quantum efficiency, namely, the number of photoelectrons traversing the GEM holes per incident $170 \mathrm{~nm}$ scintillation photon, as measured under present conditions, is only about $1 \%$. A discussion of the results is presented. (C) 1999 Elsevier Science B.V. All rights reserved.
\end{abstract}

Keywords: Scintillation counter; Gas electron multiplier; Photo electrons

\section{Introduction}

Gas proportional scintillation counters (GPSCs) are used to detect $\mathrm{X}$-rays in the $0.25-60 \mathrm{keV}$ region and have demonstrated superior performance in a number of physics and medical applications for

\footnotetext{
*Corresponding author. Tel.: + 351-39-410654;
}

which large detection areas and room temperature operation are required $[1,2]$.

In a conventional GPSC incident X-rays are absorbed in a low-electric-field drift region by the photoelectric effect in xenon. Under the influence of the electric field, the cloud of primary ionization electrons drifts into a high-field region where scintillation results from xenon excitation produced in collisions with electrons. The low-noise amplification of the primary ionization by the scintillation 
process results in a factor of two improvement in energy resolution compared with a conventional gas proportional counter [1].

A quartz-window photomultiplier tube (PMT) is presently required to detect the $170 \mathrm{~nm}$ ultraviolet (UV) scintillation photons in xenon, and this requirement has limited the widespread application of the GPSC. Quartz-window PMTs are bulky, expensive, and fragile, are sensitive to gain fluctuations, and lack the spatial uniformity necessary for large-area detectors. Furthermore, if the GPSC is operated at xenon pressures above several atmospheres, UV light losses become significant in the required thick quartz window.

Recent works have investigated integrated UV sensors as alternatives to the standard PMT $[3,4]$. In these cases, CsI was used as the photosensitive medium [5-7] and the resulting photoelectrons were charge-amplified in either microstrip or multiwire anodes operating in the pure xenon atmosphere of the GPSC. One serious drawback with these methods results from the additional scintillation photons produced in xenon during the charge avalanche process. These photons with a direct optical path to the photocathode surface can induce optical positive feedback in the system, eventually leading to electrical breakdown and a limitation on the ultimate gain that can be achieved. The gains achieved for these cases were not high enough to achieve spectroscopic performance. The design reported here attempts to circumvent this limitation by de-coupling the photocathode surface from the charge-amplification stage of the integrated photo-sensor.

This integrated photosensor can also be very attractive to other applications such as $\mathrm{RICH}$ detectors [8-10].

\section{Experimental set-up}

The design of the new integrated photosensor is schematically illustrated in Fig. 1.

The 150-nm-thick CsI photocathode is positioned $1.5 \mathrm{~m}$ from the GPSC collection grid, G2, and is vacuum-deposited onto the GEM [11-13]. The GEM (Fig. 2) is made of a 50- $\mu$ m-thick Kapton sheet coated on each side with a $70-\mu$ m-thick

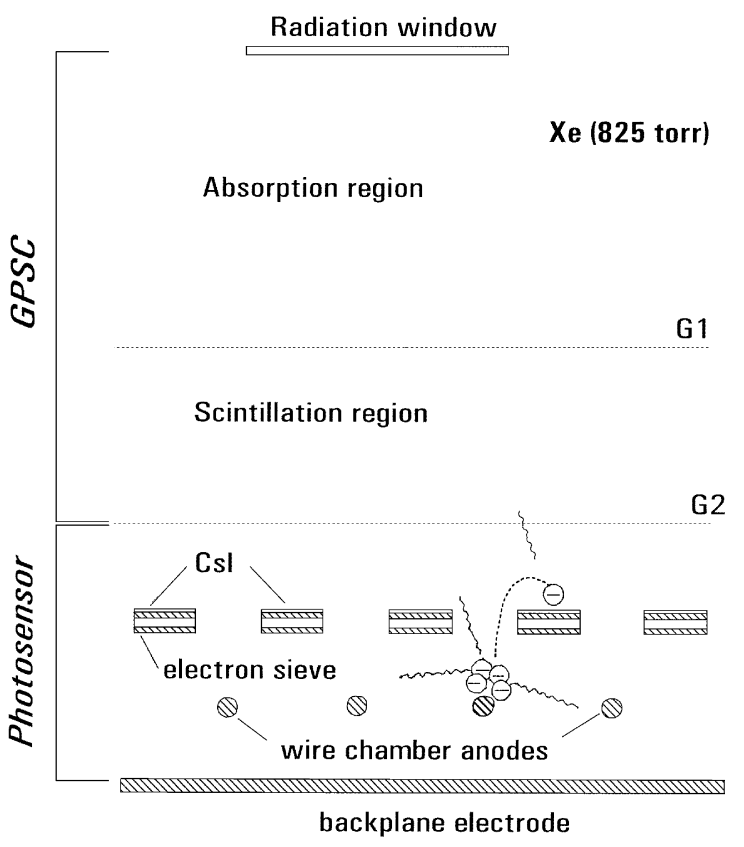

Fig. 1. Schematic diagram of the new integrated photosensor and GPSC.

vacuum-deposited copper layer and perforated with $200-\mu \mathrm{m}$-diameter holes on a $300 \mu \mathrm{m}$ pitch. The full-sensitive diameter of the GEM plate is $40 \mathrm{~mm}$, from which only $40 \%$ of the area is coated with CsI photocathode film. The remaining area corresponds to the holes in the GEM.

Directly below the GEM, a 25- $\mu$ m-diameter, 1-mm-spaced multi-wire proportional chamber anode plane functions as the charge amplification stage of the photosensor. A back-plane cathode is used beneath the multi-wire anode plane in order to guarantee electrostatic field symmetry around the multi-wire anodes. The GPSC, with a $4 \mathrm{~cm}$ deep absorption region and $1.8 \mathrm{~cm}$ scintillation region, is filled with 825 Torr of xenon, which is continuously purified through getters.

A fraction of the UV photons produced in the scintillation region (Fig. 1) illuminate the CsI and eject photoelectrons, which are accelerated along electric field lines through the holes in the GEM. The photoelectrons undergo limited charge multiplication as a result of the electric field produced by the electrodes on each side of the GEM. A much 


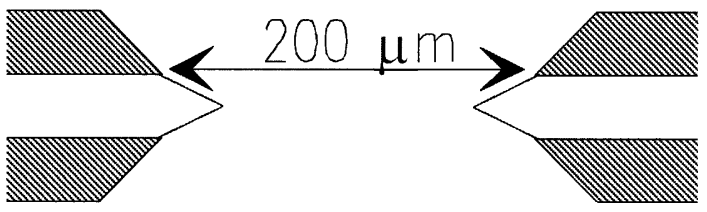

Fig. 2. Cross section of one hole of the GEM.

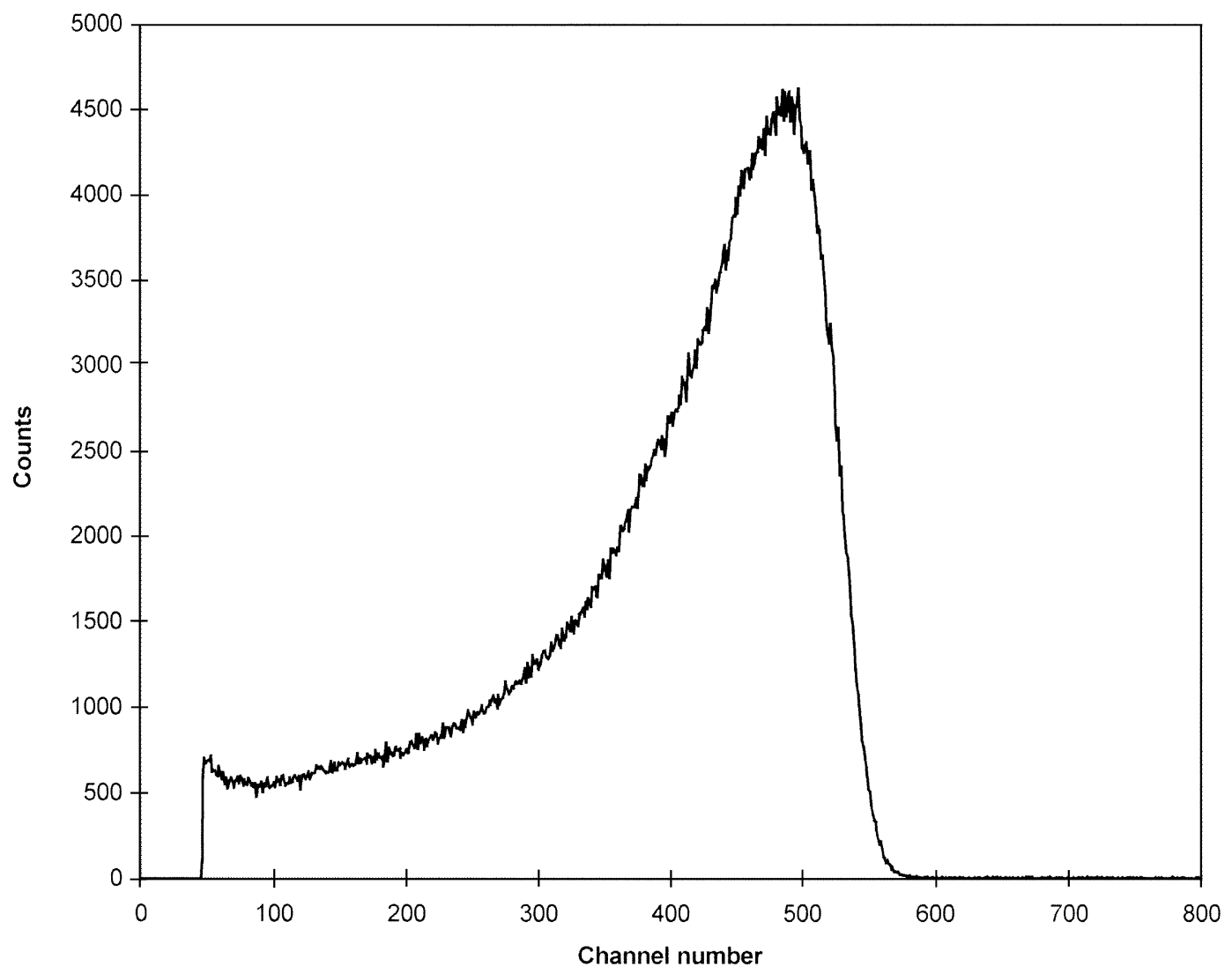

Fig. 3. Typical 5.8 MeV $\alpha$-particle spectrum obtained from the multiwire chamber anodes.

larger multiplication results from the charge avalanche around the anode wires. The additional xenon UV photons produced in the electron avalanche are effectively screened from the CsI photocathode by the intervening GEM. The amplified anode pulses depend, therefore, only on the UV light produced in the GPSC scintillation region. With this geometry it is possible, in principle, to overcome the positive feedback limitation and achieve high gains with good uniformity of response over a large detector area.

\section{Experimental results and discussion}

The pulse-height distribution for $5.8 \mathrm{MeV} \alpha$-particles from a ${ }^{241} \mathrm{Am}$ source measured from the signals taken from the wire chamber is presented in 


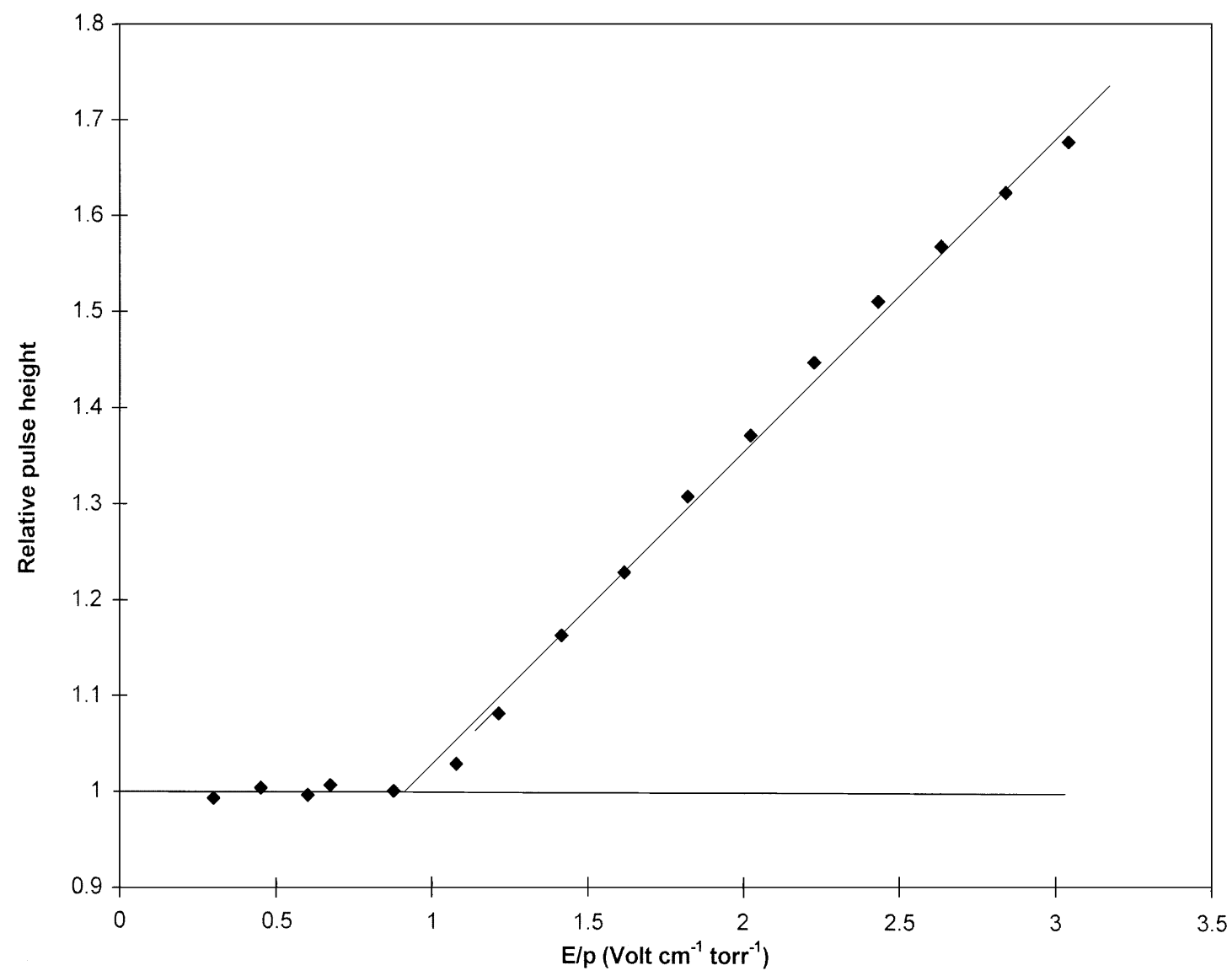

Fig. 4. Relative pulse-height amplitude as a function of the reduced electric field in the GPSC scintillation region. Full lines are the least-squares fit to the relative pulse-height amplitude for reduced electric fields below and above $1 \mathrm{~V} \mathrm{~cm}^{-1} \mathrm{Torr}^{-1}$.

Fig. 3. Since the $\alpha$-particle source is located within the xenon envelope of the GPSC with little collimation, energy resolution is deteriorated and the peak has a large, low-energy tail. No obvious peak structure was discerned for the 14- and 60-keV X-rays.

The pulse-height amplitude (Fig. 4) exhibits a straight-line dependence on the reduced electric field with a threshold at about $1 \mathrm{~V} \mathrm{~cm}^{-1} \mathrm{Torr}^{-1}$ as is the characteristic of the scintillation light from xenon [14]. This demonstrates conclusively that the pulses are produced by photoelectrons ejected from the CsI film by the scintillation photons.

In Fig. 5 we plot the charge gain of the GEM as a function of the voltage difference between its front and back surfaces. The gain was measured for the absorption of three different radiations: 14- and $60-\mathrm{keV} \mathrm{X}$-rays and 5.8 MeV $\alpha$-particles. The data for the different energies are quite similar, implying the absence of any appreciable space-charge effects. For these measurements, the voltages applied to the scintillation region were well below the scintillation threshold, so that the pulses resulted only from the collection of the primary ionization charge that has been transferred through the GEM and into the multi-wire chamber region. The charge transfer efficiency was measured to be nearly $100 \%$. Measurable charge multiplication begins at approximately $50 \mathrm{~V}$ and reaches a value of 3.3 at $250 \mathrm{~V}$. At higher voltages, instabilities attributed to the onset of breakdown occur. 


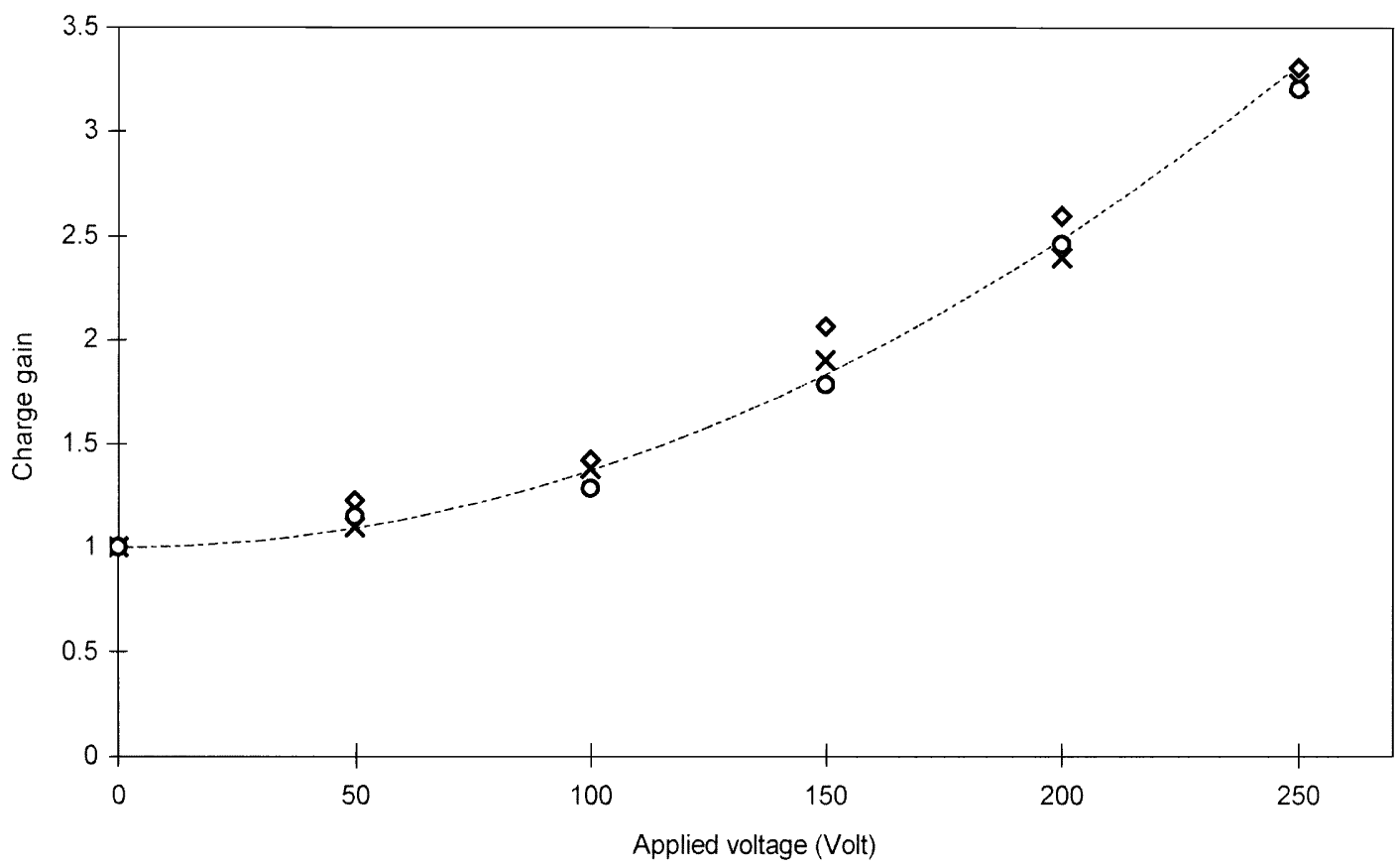

Fig. 5. GEM charge gain for $14 \mathrm{keV}(\times), 60 \mathrm{keV}(\diamond) \mathrm{X}$-rays, and $5.8-\mathrm{MeV}(\bigcirc) \alpha$-particles, as a function of the voltage between its electrode surfaces.

The photosensor multi-wire chamber charge gain as a function of the applied voltage is depicted in Fig. 6. As seen at $1100 \mathrm{~V}$ the gain is about $10^{2}$. Higher gains can be obtained but instabilities due to electrical breakdown occur.

The effective quantum efficiency, $E_{\mathrm{qe}}$, namely, the number of photoelectrons traversing the GEM per UV photon reaching the full $40 \mathrm{~mm}$ diameter photosensitive area, can be estimated form Fig. 4 from the ratio of the pulse heights with and without scintillation amplification. For $E / p$ below the scintillation threshold of $1 \mathrm{~V} \mathrm{~cm}^{-1} \mathrm{Torr}^{-1}$, the measured pulse height is due to only the primary electron number, $N_{0}$, and the detector functions as an ionization chamber. For values above $1 \mathrm{~V} \mathrm{~cm}^{-1} \mathrm{Torr}^{-1}$, in addition to $N_{0}$, we also have $N_{\text {pe }}$, the number of photoelectrons resulting from scintillation entering the holes of the GEM. With the first grid, G1, of the GPSC biased at $-5700 \mathrm{~V}$ and the second grid, G2, at $-1200 \mathrm{~V}$, the reduced electric field in the scintillation region is $3.0 \mathrm{~V} \mathrm{~cm}^{-1}$ Torr $^{-1}$, well above the scintillation threshold.
If the dependence of the pulse height on the collected charge is assumed to be linear, we can say that the pulse height is proportional to the total number of electrons that are transmitted through the GEM holes. Comparing the relative pulse heights in Fig. 4 for $E / p=3$ and $<1 \mathrm{~V} \mathrm{~cm}^{-1}$ Torr $^{-1}$, we find that

$\frac{N_{0}+N_{\mathrm{pe}}}{N_{0}}=1.70$ or $\quad N_{\mathrm{pe}}=0.70 N_{0}$.

If there is no further ionization prior to the GEM, $N_{0}$ is also the number of electrons formed when the radiation is absorbed in the GPSC. For a $5.8 \mathrm{MeV} \alpha$-particle and a mean electron/ion pair production energy in xenon of $22 \mathrm{eV}, N_{0}$ is $2.6 \times 10^{5}$.

$N_{\text {pe }}$ can also be estimated by

$N_{\mathrm{pe}}=N_{0} \times$ Light gain $\times \frac{\Omega}{4 \Pi} E_{\mathrm{qe}}$

where the light gain is the number of scintillation photons produced by one primary electron in traversing the scintillation region, $\Omega / 4 \Pi$ is the fraction of solid angle subtended by the active 


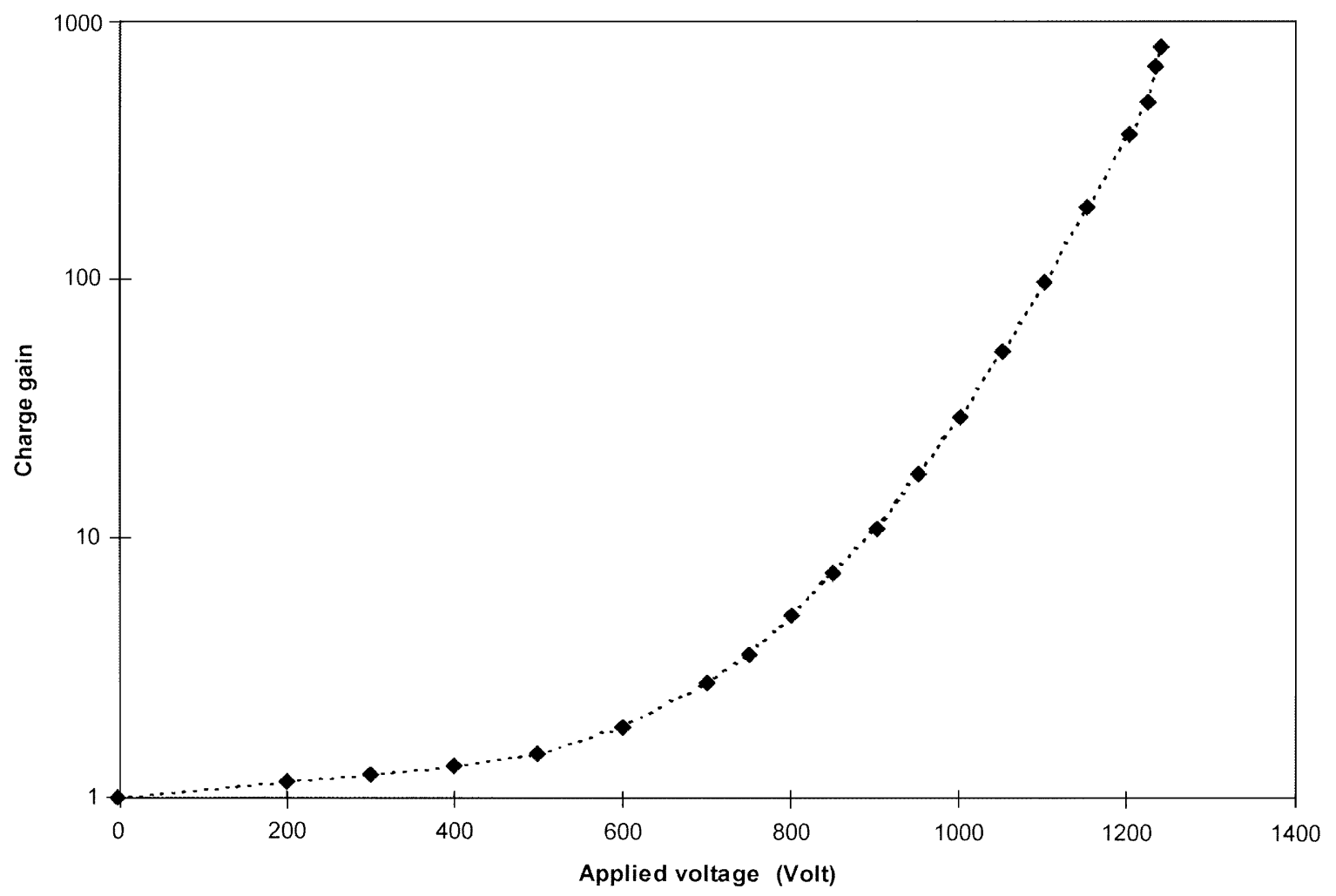

Fig. 6. Charge gain on the multi-wire chamber as a function of the applied voltage (referred to the back surface of the GEM).

photocathode area, and $E_{\mathrm{qe}}$ is the effective quantum efficiency. At $E / p=3 \mathrm{~V} \mathrm{~cm}^{-1} \mathrm{Torr}^{-1}$ in the scintillation region, the $170 \mathrm{~nm}$ scintillation photons of xenon have about $8 \mathrm{eV}$ and are produced with about $89 \%$ efficiency [15], resulting in a light gain of $4.5 \times 10^{2}$ photons/electron. $\Omega / 4 \Pi$ was calculated [16] to be $1.5 \times 10^{-1}$ for a 40 -mm-diameter GEM photosensitive area. From Eqs. (1) and (2) we can estimate the $E_{\mathrm{qe}}$ to be about $1 \%$.

Taking into account that only $40 \%$ of the GEM area is actually photosensitive (the remaining area corresponds to GEM holes), the efficiency for the actual photosensitive area is about 3\%. This efficiency is the product of the CsI quantum efficiency and the collection efficiency of the photoelectrons into the GEM. For a typical value of $30 \%$ quantum efficiency for CsI at $170 \mathrm{~nm}$ [5-7], the above estimate leads to the conclusion that only approximately $10 \%$ of the photoelectrons are transmitted through the GEM. In fact, following extraction from the CsI, the photoelectron must be directed through the GEM and toward the wire chamber. If the electric field at the photocathode surface is too weak, photoelectrons cannot be extracted from the photocathode. If the field is too strong, photoelectrons are collected on G2 instead of being transmitted through the GEM. For efficient transmission, a compromise field value has to be determined.

In Fig. 7 we plot the relative pulse height of the system as a function of the irradiation on the photosensor CsI photocathode. As seen, after the initial build-up taking place in the first $10^{5}$ photons $\mathrm{mm}^{-2}$ the system shows a very good stability, better than $\pm 2 \%$ variation on the pulse height. The initial variation in the pulse amplitude may be explained by charge build-up in the insulating holes of the GEM. 


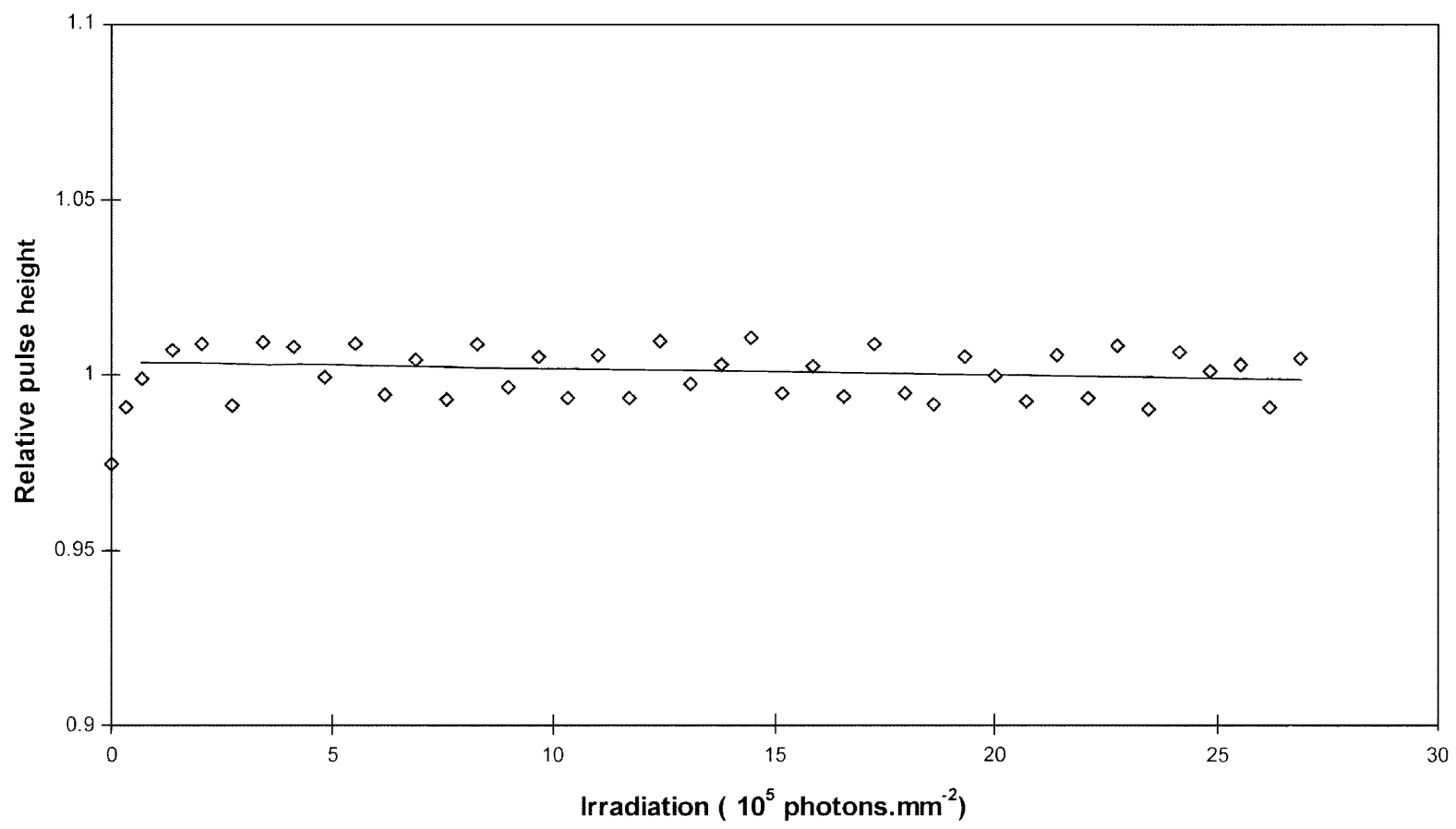

Fig. 7. Relative pulse-height amplitude as a function of the photosensor irradiation of xenon $170 \mathrm{~nm}$ scintillation light.

\section{Conclusions}

The device described here demonstrates that it is possible to detect UV scintillation light with an integrated photosensor with little or no positive feedback from photons produced in the charge avalanche process. The effective quantum efficiency presently obtained, about $1 \%$, is lower than expected. Also, electrical insulation of the multiwire chamber should be improved in order to achieve reasonable photosensor gain. These photosensors parameters limit the obtained performance of the integrated photosensor. Development work concerning the improvement of these parameters and the study of alternative geometries are in progress.

\section{Acknowledgements}

This work was carried out in the Atomic and Nuclear Instrumentation Group of the Instrumen- tation Center (Unit 217/94) of the Physics Department, University of Coimbra. It was supported by Junta Nacional de Investigação Científica e Tecnológica (JNICT) through CERN/P/FIS/1165/97.

\section{References}

[1] A. Peacock, R.D. Andresen, E.A. Leimann, A.E. Long, G. Manzo, B.G. Taylor, Nucl. Instr. and Meth. 169 (1980) 613.

[2] A. Smith, M. Bavdaz, Rev. Sci. Instr. 63 (1992) 683.

[3] J.F.C.A. Veloso, J.A.M. Lopes, J.M.F. dos Santos, C.A.N. Conde, IEEE Trans. Nucl. Sci. NS- 43 (1996) 1232.

[4] J.A.M. Lopes, J.M.F. dos Santos, C.A.N. Conde, R.E. Morgado, IEEE Trans. Nucl. Sci. NS- 44 (1997) 517.

[5] V. Dangendorf, A. Breskin, R. Chechik, H. Schmidt-Bocking, Nucl. Instr. and Meth. A 289 (1990) 322.

[6] D.F. Anderson, S. Kwan, V. Peskov, Nucl. Instr. and Meth. A 343 (1994) 109.

[7] A. Breskin, Nucl. Instr. and Meth. A 371 (1996) 116.

[8] F. Piuz, Nucl. Instr. and Meth. A 371 (1996) 96.

[9] J. Séguinot et al., Nucl. Instr. and Meth. A 371 (1996) 64. 
[10] J. Va’vra, Nucl. Instr. and Meth. A 371 (1996) 33.

[11] F. Sauli, Nucl. Instr. and Meth. A 386 (1997) 531.

[12] J. Benlloch et al., IEEE Trans. Nucl. Sci. NS-45 (1998) 234.

[13] J. Benlloch et al., Nucl. Instr. and Meth. A 419 (1998) 410 .
[14] C.A.N. Conde, L.R. Ferreira, M.F.A. Ferreira, IEEE Trans. Nucl. Sci. NS-24 (1977) 221.

[15] F.P. Santos, T.H.V.T. Dias, A.D. Stauffer, CAN Conde, J. Phys. D. 27 (1994) 42.

[16] J.M.F. dos Santos, A.C.S.M. Bento, C.A.N. Conde, IEEE Trans. Nucl. Sci. NS-39 (4) (1992) 541. 